\title{
DOSAGE DES PROTÉINES ET DES ACIDES AMINÉS ET DÉTERMINATION DE LEUR VALEUR NUTRITIVE. ( ${ }^{(1)}$
}

PAR

\author{
G. FAUCONNEA U
}

Service de Biochimie, C. N. R. Z., Jouy-en-Josas

\section{PLAN DU MÉMOIRE}

I. - Besoins azotés de l'homme et des animaux.

II. - Fraction azotée des aliments.

III. - Dosage des acides aminés totaux libres et combinés.

IV. - Valeur nutritive des protéines calculée d'après leur composition en acides aminés.

v. - La valeur nutritive réelle des protéines sur l'animal.

VI. - Corrélation des méthodes chimique et biologique de prévision de la valeur nutritive des protéines.

Devant un problème aussi vaste, nous avons dû nous limiter et nous avons insisté sur la valeur des méthodes analytiques (avec leur limite) plutôt que sur leur description technique proprement dite. En outre, nous avons abordé le problème dans le cadre général de la nutrition azotée en nous référant toujours à l'animal.

\section{I. - BESOINS AZOTÉS DE L'HOMME ET DES ANIMAUX}

On connaît l'importance des matières azotées dans la constitution et la physiologie des animaux. Les protéines constituent la plus grande partie du protoplasme des cellules en assurant leur structure. Leurs acides aminés constitutifs servent à la fabrication des enzymes et des hormones qui catalysent et règlent toutes les fonctions métaboliques tant dans la

(1) Cours-conférence, donné le 2.3 avril 1956, à la Maison de la Chimie, sous les auspices du Centre de Perfectionnement Technique. 
cellule que dans l'organisme entier : formation de tissus spécialisés (os, téguments) et protection des organismes (détoxication, anticorps). Or, les protéines en dehors du groupement prosthétique qu'elles peuvent contenir et qui concourt à leur spécificité, sont constituées de chaînes d'acides aminés (ou d'acides iminés) reliés entre eux par leur groupement carboxylique et aminé (liaison peptidique) ; les chaînes peuvent être ramifiées ou fermées, à l'aide sans doute des autres groupements polaires de certains acides aminés : hydroxyle, sulphydrile, guanidine. aminé $\beta$ ou $\gamma$ carboxyle. Ainsi 18 acides aminés de la série $I_{\uparrow}$ en proportion et en arrangements variables, assurent la formation de toutes les protéines et leur confèrent des propriétés physico-chimiques particulières.

Ces protéines sont élaborées à partir des protéines alimentaires contenant les mêmes acides aminés (avec quelques exceptions), mais en proportions différentes; ceux-ci ou leurs dérivés libérés en partie dans le tube digestif sont absorbés par la muqueuse intestinale et sont transportés par le sang jusqu'aux tissus, pour compenser leur usure (c'est le métabolisme endogène) ou assurer de nouvelles synthèses (cas de la production de la viande ou du lait). I,es proportions des acides aminés arrivant dans un lieu de synthèse peuvent être différentes des besoins pour la fabrication de telle protéine qui obéit à la loi du tout ou rien, c'est-à-dire qu'elle constituera un bloc indivis de composition constante qui sera synthétisé en totalité ou pas du tout. Il y a un équilibre dynamique entre toutes les protéines de l'organisme avec un rôle particulier du foie qui synthétise des polypeptides et les albumines; par suite des protéines seront dégradées (en totalité également) pour équilibrer l'apport d'acides aminés sur le lieu de synthèse. Ainsi, il faut apporter tous les acides aminés indispensables en même temps car l'organisme ne peut les mettre en réserve ; et le besoin azoté s'exprime par un apport équilibré de tous les acides aminés indispensables, cet apport étant chronologique par rapport aux besoins ; le caractère d'indispensabilité d'un acide aminé a lieu quand l'animal ne peut en faire la synthèse à une vitesse suffisante pour assurer les besoins; par suite l'indispensabilité varie avec la nature de ceux-ci. Quant au besoin d'azote indifférencié, il peut être assuré par un acide aminé quelconque (glycine par exemple) ou même par un sel d'ammonium (citrate d'ammonium), si les besoins énergétiques sont largement couverts.

\section{Entretien.}

Dans ce cas un acide aminé est indispensable s'il est nécessaire dans la ration pour maintenir l'équilibre azoté ou pondéral et le besoin minimum correspond à la quantité la plus faible d'acide aminé ayant permis de maintenir l'équilibre azoté sur l'individu le plus exigeant (il y a en effet 
de grandes variations individuelles pour un même poids vif). Ce besoin correspond au renouvellement de certaines protéines à l'échelon cellulaire ou organique (la classique usure des tissus), mais aussi aux exigences des fonctions de détoxication de 1'organisme : le besoin en méthionine correspondrait surtout à cette dernière hypothèse ; en effet, 1'introduction de méthionine à une ration sans azote épargne les tissus du corps (BRUsIr I947) et diminue l'excrétion de l'azote urinaire. I_a détermination des acides aminés indispensables et de l'azote indifférencié pour l'entretien de 1'homme en utilisant des rations synthétiques à base d'acides aminés purs et de sels d'ammonium ou de glycine a été effectuée par W. C. RosE (I950-I955), tableau I ; le besoin d'azote total trouvé est très bas 2,28 à $2,55 \mathrm{~g}$ suivant les individus par jour, c'est-à-dire $\mathrm{I}_{4,25}$ à $\mathrm{I}_{5}, 95 \mathrm{~g}$ de protéine pour un homme de $70 \mathrm{~kg}$. Mais ces expériences ont été effectuées dans des conditions idéales oì les acides aminés ont été apportés en quantité adéquate pour l'organisme ( 2 fois les besoins minima) et sous forme d'acides aminés libres tous utilisables en même temps. Rose n'a pas trouvé de besoin en arginine pour l'entretien de la fonction de reproduction chez l'homme contrairement aux conclusions d'ALBANISE (I942). Certains acides aminés sont dits semi-indispensables car leur présence dans la ration économise une fraction d'un acide aminé indispensable ; la cystine peut économiser $80 \mathrm{p}$. Ioo de la méthionine pour l'entretien de l'homme et la tyrosine la moitié de la phénylalamine. Pour l'entretien du rat les acides aminés indispensables sont les mêmes que ceux nécessaires à l'homme à cette différence près que le besoin en lysine est très faible (voire même inexistant).

\section{Besoins de production.}

Ces besoins s'expliquent par la composition des protéines formées (tissus musculaires dans le cas de la croissance, protéine du lait pour la lactation, protéine plasmatique) et les besoins pour la synthèse de ces différentes protéines sont voisins.

\section{Croissance.}

L,es besoins ont été déterminés sur un certain nombre d'espèces en utilisant les rations synthétiques. I,es acides aminés indispensables sont les mêmes en général que pour l'entretien; il s'y ajoute l'histidine et l'arginine pour la plupart des espèces et de plus la lysine dans le cas du rat. En outre, l'acide glutamique et la proline semblent indispensables pour obtenir la vitesse maximum de croissance. En outre, comme pour l'entretien, la cystine peut remplacer une partie de la méthionine, (40 p. Ioo,) la 


\section{TABLEAU I}

Besoins en acides aminés indispensables pour l'entretien de l'homme Rose (I950-I955)

\begin{tabular}{|c|c|c|}
\hline & $\begin{array}{l}\text { gr d'aride arniné } 2 \text { fois } \\
\text { le besoin minima }\end{array}$ & $\begin{array}{l}\text { Ngr contenu dans les } \\
\text { acides aminés }\end{array}$ \\
\hline 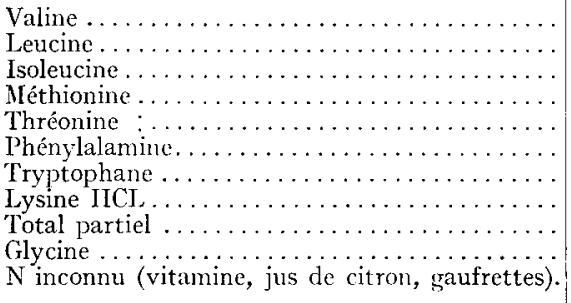 & $\begin{array}{l}3,20 \\
2,20 \\
2,80 \\
2,20 \\
2,00 \\
2,40 \\
0,50 \\
2,00 \\
6,48\end{array}$ & $\begin{array}{l}0,38 \\
0,23 \\
0,30 \\
0,21 \\
0,24 \\
0,20 \\
0,07 \\
0,31 \\
1,94(1) \\
1,21 \\
0,35\end{array}$ \\
\hline $\begin{array}{l}\text { Total } \ldots \ldots \ldots \ldots \ldots \ldots \ldots \ldots \ldots \ldots \ldots \\
\left({ }^{1}\right) \text { 1,42 I, acides aminés. } \\
0,52 \text { Isomère D Valine, Isolencine, Thréo } \\
\text { Le besoin minima se situe entre } 2,28 \text { et }\end{array}$ & $\begin{array}{l}\text { ine, Phénylalamine. } \\
55 \text { gr jar jour suivant }\end{array}$ & individus. \\
\hline
\end{tabular}

tyrosine une partie de la phénylalamine sans avoir l'une et l'autre d'utilité propre en 1'absence de l'élément indispensable qu'elles peuvent partiellement économiser. Ces acides aminés sont appelés semiindispensables. Tous les mammifères y compris l'organisme interne du ruminant (isolé de son tube digestif) semblent avoir des besoins voisins en acides aminés indispensables du moins pour la croissance (voir tableau II).

La vitesse de croissance ne dépend que du niveau d'alimentation (matière sèche consommée et énergie nette de la ration) et le besoin

TABLEAU II

Besoins en acides aminés indispensables nécessaires à la croissance de différentes espèces

Acides aminés en p. Ioo de la matière sèche de la ration

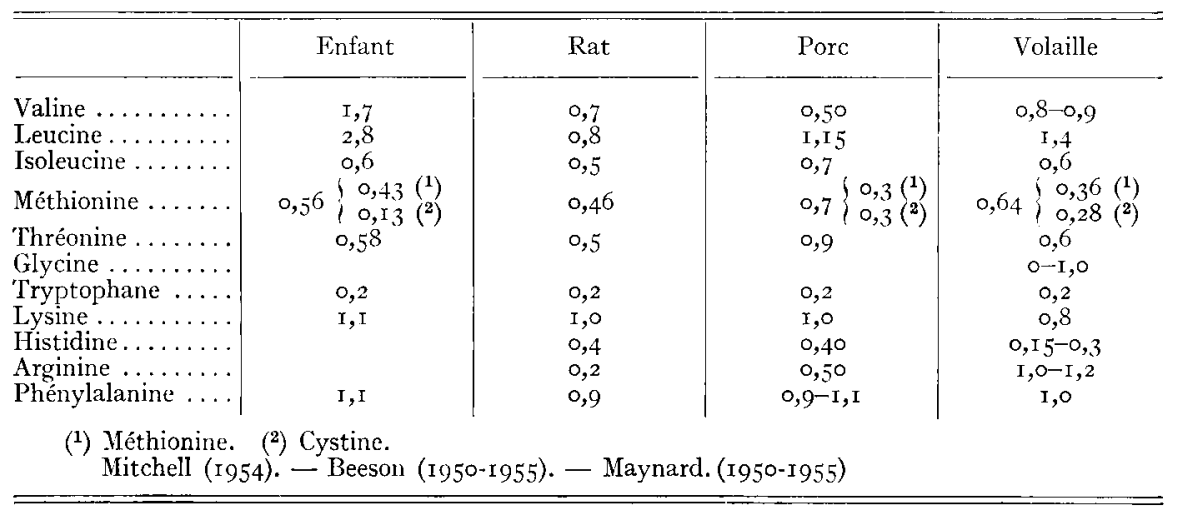


en protéine (taux protidique de la ration) est indépendant de ce même niveau (MrTcheis I953) ; aussi a-t-on exprimé les résultats en p. Ioo de la matière sèche de la ration comme le taux protidique lui-même qui varie seulement avec l'âge physiologique des animaux. En outre, comme les rations synthétiques contenant des acides aminés purs sont souvent peu appétentes, les quantités consommées et par suite les vitesses de croissance sont faibles, cependant les résultats semblent pouvoir être extrapolés comme dans le cas du taux protidique à des niveaux d'alimentation plus élevés.

En conclusion, les besoins minima en acides aminés indispensables tant pour l'entretien que pour la croissance semblent assez bien déterminés actuellement grâce à 1'utilisation de rations synthétiques pour un certain nombre d'espèces : rat, porc, volaille, homme, mais les besoins ainsi obtenus sont des besoins minima obtenus dans des conditions idéales (acides aminés indispensables apportés sous forme libre) et il est difficile de parler de besoins optimum, ou du moins de les calculer.

\section{II. - FRACTION AZOTÉE DES ALIMENTS}

On trouve différentes formes d'azote dans les aliments.

\section{Matières protéïques.}

I es protéines alimentaires sont formées de dix-huit acides $\alpha$ aminés de la série L. Elles sont généralement insolubles à 1'état natif, ou coagulables en solution dans certaines conditions (alcool 80 p. Ioo par exemple pour certaines d'entre elles).

Peptides. - Ils sont constitués d'acides aminés en nombre variable, mais plus petit que dans le cas des protéines ; ce caractère les rend précipitables ou non par certains réactifs, la solubilité dans l'eau étant cependant la règle générale; mais la séparation avec les protéines est souvent difficile à déterminer car les conditions entraînant leur précipitabilité et leur coagulabilité sont complexes.

\section{Azote non protéïque.}

I es peptides sont souvent rangés dans cette fraction qui comprend en outre - les acides aminés libres : les dix-huit acides aminés des protéines plus l'acide $\gamma$ amino-butyrique.

- les amides : asparagine et glutamine ;

- les acides aminés liés à d'autres composés: acide hippurique $=$ glycine + acide benzoïque ; 
- les bases azotées: choline, bétainne, bases puriques ;

- l'urée et l'ammoniac;

- les nitrates et les nitrites.

Au point de vue nutritionnel la séparation entre acides aminés libres, peptides et protéines n'a de sens que si la composition en acides aminés de ces fractions est différente, et la notion d'azote non protidique (préconisée par BIGWOon I953), c'est-à-dire comprenant la fraction de l'azote étrangère aux acides aminés, serait plus intéressante.

Lin sus des acides aminés usuels, les matières azotées ayant subi différents traitements (industriels par exemple) peuvent contenir des produits de transformation des acides aminés : les méthionine sulfone et sulfoxyde remplaçant partiellement la méthionine. En outre, les protéines protoplasniques de certaines cellules vivantes (bactéries) peuvent contenir l'acide $\alpha \varepsilon$ diamino pimelique.

I.e fractionnement des matières azotées d'après le caractère de solubilité ne peut être fait d'une manière générale à l'aide d'une méthode passe partout s'appliquant à tous les aliments; cependant dans certains cas, une méthode fidèle et séparant des constituants biochimiques définis peut être utilisée (appareil végétatif des plantes fourragères) mais elle ne peut être étendue à d'autres matériels sans vérification (cf. tableau III sur luzerne).

TABLEAU III

Iractions azotées de la luzerne

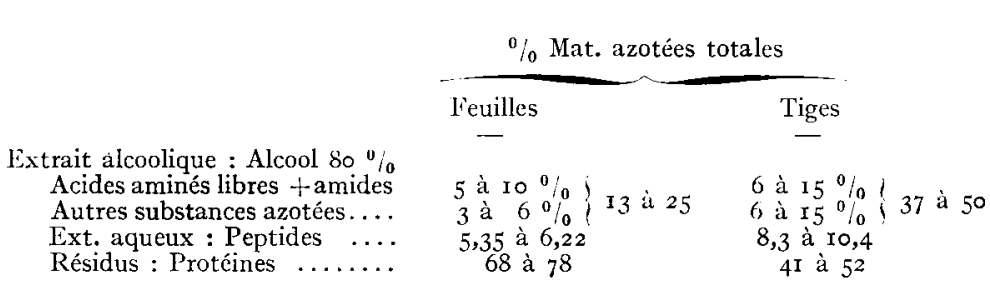

\section{III. - DOSAGE DES ACIDES AMINÉS TOTAUX LIBRES ET COMBINÉS}

I a plupart des méthodes de dosage des acides aminés exige la libération complète de ceux-ci (seuls certains acides aminés dosés par voie microbiologique et le tryptophane par voie chimique peuvent être dosés, engagés au sein de petits peptides.

\section{Hydrolyse des protéines.}

Comme la plus grande quantité des acides aminés sont engagés dans des protéines, leur dosage exige la rupture des liaisons peptidiques reliant 
les acides aminés entre eux. Comme cette liaison est assez résistante, les procédés mis en œuvre doivent être assez drastiques et peuvent ainsi modifier certains acides aminés.

Hydrolyse enzymatique. - Cette méthode est la meilleure car elle n'altère pas les acides aminés (les formes naturelles $\mathrm{L}$, sont conservées), cependant comme elle n'est jamais complète in vitro même par l'action successive et prolongée de plusieurs enzymes, elle n'est employée que pour le dosage du tryptophane par voie microbiologique. Cette méthode peut aussi être utilisée pour tester in vitro la facilité de libération des acides aminés dans les produits ayant subi certains traitements industriels (voir plus loin dans le texte).

Hydrolyse acide. - On utilise la plupart du temps l'action de 1'acide sulfurique ou chlorhydrique six fois normal pendant I 8 à 24 heures à IIO- $120^{\circ}$; le rapport poids de protéines/volume de 1'acide varie entre I/20 et $\mathrm{I} / 200$. Au cours de l'hydrolyse les acides aminés sont libérés et racémisés plus ou moins complètement : une partie des acides aminés sous forme $\mathrm{I}$, se transforment en forme D jusqu'à obtenir l'égalité entre les deux formes. Certains acides aminés en présence de glucides sont détruits complètement (le tryptophane), d'autres partiellement (arginine, histidine, cystine et méthionine). Ces destructions sont dues à une réaction entre le groupement aminé des acides aminés et le groupement aldéhydique des sucres (réaction de MAILLARD). En milieu réducteur et en l'absence des ions métalliques, la formation des produits colorés (mélanoïdines et matières humiques) résultant de la réaction précédente est réduite : 1'hydrolyse sous azote avec de 1'acide chlorhydrique très pur associé quelquefois à des réducteurs (étain chlorure stanneux) diminue les destructions ; mais il faut signaler que ces dernières sont plus importantes quand les acides aminés sont engagés en liaison peptidique.

L'acide chlorhydrique est éliminé par distillation sous vide et est employé de préférence à l'acide sulfurique difficile à éliminer sous forme de sulfate de baryum sans entraînement d'acides aminés.

Hydrolyse alcaline. - L'emploi de la soude 5 fois normale pendant 5 heures à $\mathrm{IIO}^{\circ}$ provoque une caramélisation intense des sucres et une racémisation totale des acides aminés avec destruction de beaucoup d'entre eux : arginine, histidine, cystine, méthionine; seul le tryptophane est peu détruit, il est isolé sous forme de peptides mercuriques et dosé par voie chimique.

L'hydrolyse des produits végétaux riches en glucides divers est particulièrement délicate et certains auteurs ont travaillé à l'aide de protéines extraites purifiées. Comme la valeur représentative de celle-ci est souvent douteuse, beaucoup travaillent actuellement sur le produit brut en prenant certaines précautions pour minimiser les pertes de cer- 
tains acides aminés particulièrement labiles (grand volume d'acides par rapport à la protéine hydrolysée - milieu réducteur). Le test de recouvrement d'une surcharge en acides aminés est nécessaire mais non suffisant pour juger une méthode d'hydrolyse, car la labilité des acides aminés est souvent plus grande en liaison peptidique. Cependant, sauf pour la cystine les pertes au cours de l'hydrolyse ne doivent pas excéder Io p. Ioo.

\section{Dosage des acides aminés totaux.}

L,es méthodes employées visent à doser la fonction $\alpha$ aminée primaire libre à l'exclusion des peptides et des composés azotés non aminés.

\section{Méthodes gazométriques de Van Slyke à l'acide nitreux.}

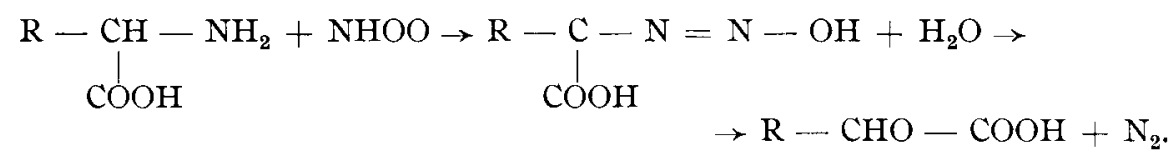

La formation d'un nitrile instable donne de l'azote gazeux et le dosage manométrique de ce gaz dégagé est une mesure de la fonction amine primaire aliphatique en général car les amines et l'ammoniac réagissent de la même façon mais moins rapidement ; pendant les 2 à 4 minutes de la réaction (fonction de la température et du volume) $25 \mathrm{p}$. Ioo de l'ammoniac et 6,I p. Ioo de l'urée réagissent seulement.

Méthode gazométrique de Van Slyke à la ninhydrine.

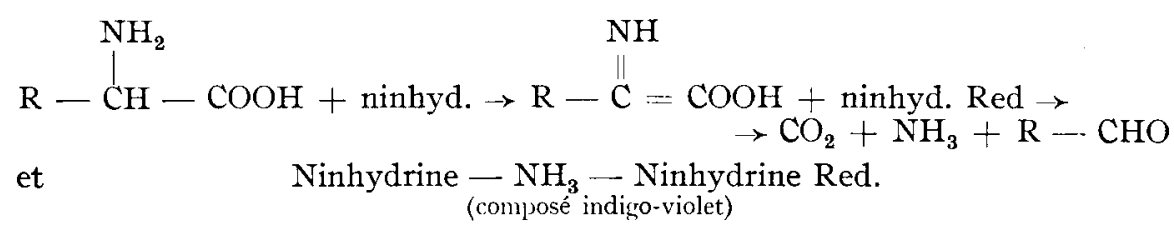

Cette méthode est un peu plus spécifique de la fonction $\alpha$ aminée car le dosage manométrique porte sur le $\mathrm{CO}_{2}$ et donne une mesure des seuls groupements carboxyles libres, attachés au même atome de carbone que ceux portant la fonction $\alpha$ aminé libre. Elle ne dose pratiquement que les acides aminés libres à l'exclusion de la plupart des peptides et des autres substances azotées.

Ces méthodes très sensibles dans leur version manométrique permettent de doser de 50 à I ooo Y d'azote $\alpha$ aminé avec une fidélité de 2 à 3 p. Ioo. Elles sont couramment utilisées pour juger les cinétiques d'hydrolyse et les recouvrements d'acides aminés après différents traitements. 


\section{Dosage des acides aminés individuels}

Méthodes chimiques. - Celles-ci employant la colorimétrie ou les précipitations fractionnées ont été exclusivement utilisées jusqu'en I944, elles manquent en général de spécificité et souvent de sensibilité, aussi exigent-elles que - les prises d'essai destinées au dosage contiennent des quantités relativement grandes de l'acide aminé à doser 50 à $500 \gamma$ (facile à réaliser dans les protéines purifiées); d'autre part, elles exigent un fractionnement préalable des hydrolysats par ionophorèse on chromatographie sur colonne : bases hexoniques, di-acides, acides aminés à noyau aromatique et acides aminés neutres monoaminés et monocarboxyliques. Ces méthodes en général s'appliquent aux protéines purifiées et il est indispensable d'extraire préalablement les protéines des aliments d'origine végétale particulièrement pauvres en azote et riches en glucides divers (grains et fourrage). Comme la valeur représentative des protéines extraites laisse à désirer, ces méthodes tendent à être abandonnées du moins pour le dosage de tous les acides aminés indispensables dans les aliments les plus pauvres en matière azotée.

Beaucoup de résultats des tables de BLOck et Bolling ont été obtenus par ces méthodes de spécificité médiocre; qui peuvent cependant encore être employées pour certains acides aminés particuliers (proline et tryptophane).

Méthodes microbiologiques. - Celles-ci datent de I943; elles utilisent la propriété qu'ont certaines bactéries lactiques (homo et hétérofermentaires) d'exiger certains acides aminés pour leur croissance. Ce caractère d'indispensabilité de l'acide aminé pour les souches utilisées est une condition nécessaire mais non suffisante pour l'emploi de celles-ci ; le milieu doit apporter tous les éléments nécessaires et en quantités équilibrées pour la croissance du nicroorganisme sauf celui à doser ; de plus, le milieu ne doit pas être sensible à l'apport des constituants de l'échantillon (autres que l'élément à doser). On mesure en général la croissance maximum de la population bactérienne car les corps interférents agissent plus sur la vitesse de croissance que sur la croissance maximum obtenue ; le temps nécessaire varie d'ailleurs avec les souches et les acides aminés à doser. On utilise comme test de croissance l'opacité de la suspension bactérienne à la fin de la phase logarithmique ou mieux la quantité d'acides formés (lactique principalement) pendant la phase fermentaire à partir du glucose ; cette dernière mesure s'effectue en général par titrimétrie.

Pratiquement on étudie parallèlement la croissance de la population bactérienne pour différentes concentrations tant de l'acide aminé pur que de l'échantillon à analyser, et la superposition des deux courbes (échantillon et standard) est une condition nécessaire pour la validité des résultats obtenus. 
Ces méthodes sont très sensibles car elles permettent de doser de I à $50 \gamma$ d'acides aminés ; leur spécificité est bonne, car elles permettent de différencier les isomères optiques $\mathrm{D}$ et $\mathrm{L}$; cependant, quelquefois les peptides peuvent réagir plus que l'acide aminé qu'ils contiennent; et dans le cas où les peptides réagissent comme l'acide aminé contenu, une hydrolyse incomplète peut être effectuée ce qui diminue les risques de pertes. La fidélité de la méthode est de plus ou moins 5 p. Ioo environ. Enfin, ces méthodes peuvent être appliquées sur l'hydrolysat acide sans fractionnement préalable et sans dessalification. Ces méthodes sont cependant délicates car elles exigent un milieu contenant 40 éléments très purs (acides aminés, bases azotées, vitamines) ; et de nombreux corps interférents peuvent exister dans les aliments ou apparaître au cours de l'hydrolyse : ils provoquent une inhibition ou une exaltation de la croissance du microorganisme sans action de croissance propre en l'absence de l'acide aminé à doser.

L'application de ces méthodes utilisables pour tous les acides aminés a permis d'établir pour la première fois des relevés relativement complets de la composition en acides aminés des milieux complexes naturels constitués par les aliments. I,es acides aminés sont dosés directement dans les hydrolysats acides obtenus par hydrolyse du produit originel sans extraction préalable de protéines. Ces méthodes sont particulièrement indiquées pour le dosage de certains acides aminés indispensables (acides aminés soufrés) où les autres méthodes sont encore plus imparfaites.

Méthodes utilisant les décarboxylases bactériennes. - On utilise les décarboxylases spécifiques de certains acides aminés (lysine, arginine, histidine, tyrosine, ornithine, acide glutamique); ces décarboxylases sont isolées dẹ cultures de certaines bactéries après action de l'éther et de 1'acétone; 1'anhydride carbonique dégagé est mesuré au Warburg. Ces méthodes sont assez spécifiques et fidèles, mais elles sont longues et ne permettent pas de doser tous les acides aminés indispensables.

Chromatographie sur papier. - Cette méthode est extrêmement séduisante ; développée après I948, elle a permis des analyses qualitatives rapides et faciles. Un spot contenant un mélange d'acides aminés dépourvus de sels ( 2 à ro $\gamma$ de chaque) est déposé au bord d'une feuille de papier filtre située dans une enceinte fermée ; le papier se sature d'eau (Io à I 5 p. Ioo de son poids) puis du solvant préalablement saturé d'eau acidifié ou non (phénol-butanol) parcourt le papier et entraîne les acides aminés à des vitesses différentes suivant leur solubilité relative dans l'eau (ou plutôt le complexe papier-eau) et le solvant. La séparation complète des 18 acides aminés exige l'emploi de 2 solvants parcourant le papier dans 2 directions perpendiculaires (phénol-eau-cupron, butanolacide acétique-eau). Le rendement quantitatif des chromatogrammes 
n'a lieu que pour des quantités faibles d'acides aminés (inférieures à Io $\gamma$ ) ; les acides aminés séparés dans ces conditions peuvent être dosés sur le papier grâce à la ninhydrine à condition que cette réaction soit quantitative, ce qui exige : l'anaérobiose réalisée par l'atmosphère d'anhydride carbonique - température constante - atmosphère d'alcool. La plupart des acides aminés réagissent quantitativement sur le papier et la coloration obtenue peut être éluée et mesurée au spectrophotomètre. Seuls les acides aminés soufrés, la proline et le tryptophane ne peuvent pas être dosés par cette méthode dont la fidélité est environ plus ou moins Io $\mathrm{p}$. Ioo. Cette méthode relativement rapide n'exige pas de très gros moyens; les échantillons hydrolysés doivent être complètement dessalifiés sans pertes d'acides aminés avant leur utilisation pour la chromatographie quantitative; on enploie à cet effet une chromatographie préalable sur colonne d'échangeur de cation (Dowex 50 ou permutite 50) mais le recouvrement de certains aminés n'est pas quantitatif (arginine).

Chromatographie sur colonne. - (DOWEX 50, MOORE et STEIN I95I). $I_{1}$ es acides aminés sont absorbés sur une colonne de polystyrène sulfoné possédant des groupements polaires sous forme $\mathrm{SO}_{3}$ - ; les acides aminés sont absorbés par leur groupement $\mathrm{NH}_{3}{ }^{+}$puis élués par des tampons de $\mathrm{pH}$ croissant. I a colonne contient le Dowex 50; l'échantillon ou le même standard d'acides aminés est mis sur la colonne, puis des tampons traversent celle-ci entraînant petit à petit les acides aminés; les gouttes sortant de la colonne sont recueillies par fraction de $\mathrm{I} \mathrm{cm}^{3}$ grâce à un dispositif adéquat (siphon par exemple) ; les tubes se déplacent automatiquement puis le réactif nécessaire pour que la réaction à la ninhydrine s'effectue est introduit : les premières fractions ne développent pas de coloration, puis les suivantes en développent une de façon croissante, passent par un maximum pour décroître ensuite. Ce groupe de tubes constitue le premier acide aminé élué. Un dosage spectrophotométrique de la coloration des différents tubes est effectué et la somme des résultats obtenus dans le groupe de tubes contenant un seul et même acide aminé donne le taux de celui-ci dans la prise d'essai introduite sur la colonne.

Comme le débit est de $4 \mathrm{~cm}^{3}$ par heure et qu'il faut $600 \mathrm{~cm}^{3}$ pour obtenir tous les acides aminés, la méthode permet d'obtenir tous les acides aminés en une semaine avec une fidélité de plus ou moins 5 p. Ioo sauf pour le tryptophane, la cystine et la méthionine. Cette méthode paraît être la meilleure actuellement, mais elle est particulièrement exigeante en travail et en matériel ; elle a fourni ces deux dernières années les analyses complètes d'un certain nombre d'aliments, celles-ci sont surtout effectuées en Belgique sous l'impulsion du Professeur BIGWood.

E,rl conclusion, la plupart des acides aminés peuvent être dosés par différentes méthodes, microbiologiques et chromatographiques ; la com- 
paraison des résultats obtenus par ces différentes méthodes pourra seule fournir des résultats valables, pour la plupart des acides aminés indispensables ou non ; seuls sont non vérifiables. les résultats des acides aminés soufrés dosables uniquement par voie microbiologique.

Limite des méthodes d'analyses et valeur des résultats obtenus. L'examen des résultats publiés par BLock et BoLLING montre des variations importantes pour un même échantillon analysé par différents laboratoires, de plus certains résultats sont systématiquement faux : la lysine obtenue par précipitation ou par différence après séparation ionophorétique des bases hexoniques procure des résultats trop faibles. Si on admet plus ou moins 5 p. Ioo comme fidélité de la plupart des méthodes utilisées chimique et microbiologique, dans un même laboratoire, les différences observées sont beaucoup plus importantes entre laboratoires. Aussi en I946, le Bureau de Recherches Biologiques de 1'Université Rutgers a fait des comparaisons entre les résultats obtenus par différents laboratoires pour l'analyse des acides aminés. Les dosages ont été effectués de 1947 à I950 par voie chimique après fractionnement préalable et par voie microbiologique, II laboratoires ont participé à ce travail qui comportait l'analyse d'un mélange standard et de 6 protéines dont les échantillons hydrolysés et bruts ont été envoyés aux différents laboratoires qui ont effectué le dosage en même temps. De grandes variations sont observées dans les résultats des différents laboratoires même pour l'analyse du mélange standard : les chiffres extrêmes de recouvrement varient pour la cystine de -22 à $+7 \mathrm{I}$ p. IOo, pour le tryptophane de -37 à +23 p. Ioo et pour la lysine de -22 à + I6 p. Ioo. Dans l'analyse des protéines les variations étaient encore plus considérables : les résultats du tryptophane varient de I à 3 pour certaines d'entre elles (farine d'arachide, gluten de blé) cependant que ceux de la cystine varient de I à 2,5 et ceux de la lysine de I à 2. I,es variations provenant des différentes techniques d'hydrolyse sont négligeables vis-à-vis des autres causes d'erreur intrinsèques. Aucune conclusion n'a pu être tirée quant au mérite relatif des méthodes chimique et microbiologique esssayées. Les résultats retenus sont des valeurs sélectionnées après élimination des résultats fournis par certains laboratoires (mauvais recouvrement des acides aminés du mélange standard).

\section{IV. - VALEUR NUTRITIVE DES PROT EINES CALCULÉE D'APRES LEUR COMPOSITION EN ACIDES AMINÉS}

Connaissant les besoins, la détermination quantitative des acides aminés indispensables, permet théoriquement de prévoir la valeur nutritive d'une protéine à condition que la digestibilité soit bonne, c'est-à-dire que les acides aminés arrivent effectivement et en même temps dans le 
sang. On prend comme terme de comparaison la protéine ou le mélange de protéines connu comme le plus utilisable tant au point de vue digestif que métabolique : les protéines de l'œuf remplissent ces conditions tant pour la croissance du rat que pour l'entretien de l'homme. Les protéines totales de 1'œuf étant le mélange protéique le mieux équilibré, on compare la composition d'une protéine donnée à celle de l'œuf quant aux acides aminés indispensables. On appelle facteur limitant 1'acide aminé indispensable dont la déficience est la plus grande (par rapport aux protéines de l'œuf), car il entraîne la valeur partielle de l'aliment. Mitchel, et BLock (I946) ont pensé que le pourcentage de déficit de ce facteur permettrait d'apprécier assez exactement la valeur nutritive d'une protéine : l'index chimique d'une protéine est ainsi calculé en soustrayant de roo le pourcentage de déficit du facteur limitant par rapport aux protéines totales de l'ouf. OSER I95I a proposé également un index d'acide aminé essentiel pour comparer les protéines aux protéines totales de l'œuf ; celui-ci est la moyenne géométrique des pourcentages de chaque acide aminé dans la protéine par rapport au contenu correspondant dans les protéines totale de 1'œuf; les pourcentages supérieurs à roo sont considérés comme roo et les pourcentages o comme $r$. Ces deux méthodes foumissent des index voisins et pour $3 \mathrm{I}$ protéines, le coefficient de corrélation entre les deux index est de 0.95 . Une autre méthode a été proposée par les auteurs

\section{TABLEaU IV}

Composition en acides aminés de quelques protéines (Valeur sélectionnée en p. Ioo des matières azotées supposées i6 p. Ioo d'azote)

\begin{tabular}{|c|c|c|c|c|c|c|c|}
\hline . & $\begin{array}{l}\text { Farine } \\
\text { de sang }\end{array}$ & $\begin{array}{l}\text { Lait } \\
\text { écrémé } \\
\text { sec }\end{array}$ & $\begin{array}{c}\text { Muscle } \\
\text { de bœuf } \\
\text { (Rutgers) }\end{array}$ & $\begin{array}{c}\text { (Euf } \\
\text { entier } \\
\text { (Rutgers) }\end{array}$ & $\begin{array}{c}\text { Blanc } \\
\text { d'œuf } \\
\text { (Rutgers) }\end{array}$ & $\begin{array}{c}\text { Farine } \\
\text { d'arachide } \\
\text { (Rutgers) }\end{array}$ & $\begin{array}{l}\text { Gluten } \\
\text { de blé } \\
\text { (Rutgers) }\end{array}$ \\
\hline Tyrosine. & 2,64 & 4,09 & 2,85 & $.3,63$ & 3,02 & 3,28 & 2,14 \\
\hline Arginine & 4,34 & 3,15 & 6,88 & 6,49 & 5,93 & $12,3^{8}$ & 3,76 \\
\hline Histidine. & 6,28 & $2,+5$ & 3,43 & 2,07 & 2,45 & 2,23 & $2, \mathrm{I} \mathrm{I}$ \\
\hline Isoleucine. & 1,52 & 6,97 & 5,3 & 5,67 & 6,02 & 3,89 & 4,26 \\
\hline Leucine . & 10,10 & 9,85 & 8,14 & 8,78 & 8,65 & $7, \mathrm{II}$ & 6,98 \\
\hline l.ysine. & 9,12 & 8,42 & 10,13 & 7,24 & 7,66 & 4,53 & 2,10 \\
\hline léthionine ..... & $\mathrm{I}, \mathrm{I} 7$ & $2,0.3$ & 2,58 & 3,85 & $3,5^{8}$ & 0,71 & $r, 60$ \\
\hline Phénylaladine .... & 7,01 & 4,45 & 4,38 & $5,7 \mathrm{I}$ & 6,49 & $5,4 \mathrm{I}$ & $5,5.3$ \\
\hline Thréonine .............. & $4,5 \mathrm{I}$ & 4,30 & 5,06 & 5,29 & 4,91 & 2,84 & 2,80 \\
\hline Valine & 8,59 & 6,33 & 6,97 & 8,79 & 9,30 & $5,5^{\circ}$ & 5,34 \\
\hline Tryptophane & $\mathrm{r}, 35$ & 1,18 & $\mathrm{I}, 06$ & $\mathrm{I}, 3 \mathrm{I}$ & 1,38 & 0,92 & $0,7^{6}$ \\
\hline
\end{tabular}

Williams (r955) - Coopérative détermination Rutgers Univers (r950).

allemands KüNHAU et NEHRING en prenant comme référence les protéines du lait humain ; celle-ci est destinée à la prévision de la valeur nutritive des protéines utilisées en nutrition humaine (voir tableau V).

L'index chimique de MrTCHELL et BLOCK mesure la valeur nutritive des protéines destinées à la croissance seule, car il suppose que le facteur 


\section{TABLEAU V \\ Valeur nutritive des protéines}

calculées d'après leur composition en acides aminés

Index chimique :

(Mitcinell, I946) $=$ I00 - \% de déficit des facteurs limitants. - lix. gluten de blé $\cdot$ facteur imitant : Lysine. $-\%$ de déficit $\frac{7,24-2,10}{7,24} \times 100=7 \mathrm{r} \%$. Index chimique $=29$.

Index d'acide aminé essentiel : (OSER, I95I).

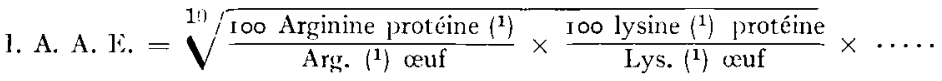

ou $\log =\frac{I}{10}\left(\log \frac{100 \text { Arg. prot. }}{\text { Arg. ouf }}+\log \frac{\text { Lysine prot. }}{\text { Lyisne weuf }}+\cdots\right)$.

(1) Exprimé en \% des matières azotées supposées à $16 \%$ d'azote).

limitant conditionne seul l'utilisation des autres acides aminés indispensables présents dans les protéines. Quant à l'entretien comme nous l'avons dit à propos des besoins, certains acides aminés et par suite des protéines incomplètes peuvent diminuer les pertes d'azote endogènes, et être ainsi utilisées valablement pour l'entretien ; dans ce cas, 1'index d'OSER apprécie mieux la valeur nutritive des protéines, ce qui est valable également pour la croissance, puisque cette dernière ne peut être étudiée isolément.

\section{V. - LA VALEUR NUTRITIVE RÉELLE DES PROTÉINES SUR L'ANIMAL}

Comme les besoins varient avec les états physiologiques, productions et entretien, la notion de valeur nutritive est relative, aussi faut-il d'abord analyser les facteurs d'utilisation et aborder ensuite les méthodes utilisées pour apprécier sur l'animal la valeur nutritive réelle des protéines en vue d'assurer les différents besoins.

Digestibilité. - L'utilisation digestive se caractérise par le coefficient de digestibilité apparent ou réel

$$
\begin{aligned}
& \text { CUDA }=\frac{\mathrm{Ni}-\mathrm{Nf}}{\mathrm{Ni}}{ }^{(1)} \\
& \text { CUDR }=\frac{\mathrm{Ni}-(\mathrm{Nf}-\mathrm{Nmf})}{\mathrm{Ni}}{ }^{(1)}
\end{aligned}
$$

ce dernier est plus rigoureux car il élimine de l'azote fécal (Nf) la fraction endogène appelée azote métabolique fécal ( $\mathrm{Nmf}$ ), qui est obtenu pendant une période de jeune, protidique, l'animal conservant le même niveau d'alimentation.

(1) CUDA $=$ coefficient d'utilisation digestive apparent.

CUDR $=$ coefficient d'utilisation digestive réel.

$\mathrm{Ni}=$ azote ingéré.

$\mathrm{Nf}=$ azote fécal.

Nmf = azote métabolique fécal. 
En réalité le coefficient d'utilisation digestive réel est voisin de Ioo p. Ioo pour la plupart des protéines des aliments naturels et les coefficients de digestibilité différents ne sont qu'une conséquence de l'effet de l'azote métabolique fécal relativement constant sur des rations de taux protidiques variables, et le coefficient de digestibilité apparent augmente régulièrement avec le taux protidique (variance contrôlée 73 p. roo) CRAMPTON I954.

Métabolisation. - I'utilisation métabolique se caractérise classiquement par la mesure de la valeur biologique; et celle-ci varie avec les différents états physiologiques des animaux.

Entretien. - I a valeur biologique permet d'apprécier la valeur nutritive des protéines pour l'entretien à condition d'opérer dans des conditions bien standardisées : taux protidique minimum correspondant à des bilans azoté et pondéral nuls.

$$
\text { V. B. }=\frac{\mathrm{Ni}-(\mathrm{Nf}-\mathrm{Nmf})-(\mathrm{Nu}-\mathrm{Nue})}{\mathrm{Ni}-(\mathrm{Nf}-\mathrm{Nmf})} .
$$

I a difficulté réside dans l'appréciation de l'azote métabolique fécal et de l'azote urinaire endogène ; car, ces déterminations opérées pendant une période de jeûne protéique avec le même niveau de nutrition, supposent l'indépendance absolue des métabolismes endogènes et exogènes qui est une conception assez relative (BRUSH 1947).

Croissance. - Pour cette dernière comprenant l'entretien et la croissance, la formule a été adaptée par Mitchfi,

$$
\text { V. B. }=\frac{N \text { Bilan }+N u e+N m f}{N i-(N f-N m f)}
$$

mais 1'application est particulièrement difficile car beaucoup de facteurs influent sur elle et peuvent la modifier. Si le taux protidique de la ration est tel que le besoin en acides aminés des tissus est plus grand que l'apport, la valeur biologique (pour la croissance) sera alors maximum, mais dans ces conditions une déficience relative en protéine existe et la croissance obtenue n'est pas maximum.

Cette notion a même été appliquée à la production du lait par MORRIS.

$$
\mathrm{V} . \mathrm{B} .=\frac{\mathrm{N} \text { du lait }}{\mathrm{N} \text { total assimilé }-\mathrm{N} \text { assimilé nécessaire à l'éntretien }} .
$$

Vu les difficultés de mesure de la valeur biologique, d'autres méthodes ont été proposées pour tester la valeur nutritive des protéines.

Efficacité protéique. — C'est le gain de poids en gramme rapporté

(1) $\mathrm{Nu}=$ azote urinaire

Nue $=$ azote urinaire endogène. 
au gramme de protéines ingérées, les mesures étant effectuées sur le rat. Cette mesure tient compte de la digestibilité et de la métabolisation et elle est un effet global de la protéine sur l'animal.

Déplètion. - L'aptitude des protéines à la reconstitution des tissus chez 1'adulte peut être testée par la méthode de la déplètion : 1'animal est soumis au jeûne jusqu'à perte de $25 \mathrm{p}$. Ioo de son poids, puis on mesure comme pour l'animal en croissance le gain de poids rapporté au gramme de protéines ingérées. Si l'on veut mesurer l'aptitude à la reconstitution des protéines plasmatiques et globulaires, une saignée effectuée en fin de période de déplètion permet de mesurer la reconstitution de celle-ci pendant la période de réalimentation. Ces méthodes mesurent l'effet global sur l'animal en faisant intervenir tous les facteurs de 1'utilisation alors que la valeur biologique ne teste souvent que la métabolisation.

\section{VI. - CORRÉLATION DES MÉTHODES CHIMIQUE ET BIOLOGIQUE DE PRÉVISION DE LA VALEUR NUTRITIVE DES PROTÉINES}

Dans quelle mesure l'analyse des acides aminés indispensables permet-elle de prévoir la valeur nutritive réelle pour l'animal ?

\section{Monogastriques.}

Entretien. - Le dosage quantitatif des acides aminés indispensables à l'entretien contenus dans une protéine permet la prévision de la valeur nutritive de celle-ci. De nombreuses expériences ont été effectuées : le rat a un besoin très faible de lysine pour son entretien, aussi les aliments pauvres en cet acide aminé (gluten de blé et farine blanche) ne sont pas améliorés par 1'adjonction de lysine, et la valeur biologique mesurée pour l'entretien du rat est plus élevée que celle obtenue pour l'entretien de l'homme, lequel exige la lysine comme acide aminé indispensable.

Le Bureau de Recherches Biologiques de l'Université Rutgers a fait effectuer corrélativement aux analyses d'acides aminés mentionnées plus haut, de nombreuses déterminations de la valeur nutritive des six protéines étudiées à l'aide de tous les tests mentionnés plus haut, tant pour l'entretien et la croissance, que pour la production laitière et les fonctions de reproduction. Vingt laboratoires différents ont utilisé ces méthodes standardisées sur différentes espèces : rat, souris, chiens, homme (voir tableau V).

La valeur nutritive des protéines pour l'homme adulte et pour le rat en croissance, sont très voisines ; la corrélation entre les valeurs biologiques est de 0,9I5 tandis qu'elle n'est que de 0,670 entre les valeurs 
biologiques pour le rat adulte et l'homme adulte. Par suite, la valeur nutritive des protéines pour l'homme adulte peut être prévue valablement par la mesure de l'efficacité protéique sur le rat en croissance. Enfin Bocolo (I952) a signalé la corrélation étroite existant entre la composition en acides aminés de l'œuf entier et celle de différents tissus humains (muscles, foies, cœurs) $\mathrm{R}=0,8 \mathrm{II}$; de plus le coefficient de corrélation de la composition des tissus humains avec différents aliments diminue comme leur valeur biologique.

$$
\begin{aligned}
& \mathrm{R}=0,79 \mathrm{I} \text { pour la caséine } ; \\
& \mathrm{R}=0,5 \mathrm{I} 6 \text { pour le gluten } \\
& \mathrm{R}=0,455 \text { pour le maïs entier. }
\end{aligned}
$$

Croissance. - Nous mentionnerons encore les résultats obtenus par le Bureau de Recherches Biologiques de l'Université Rutgers, car les teneurs en acides aminés sont des valeurs sélectionnées parmi les laboratoires ayant obtenu les meilleurs résultats de recouvrement du mélange standard. Pour la croissance du rat l'efficacité protéique mesurée par 7 laboratoires permet de classer les aliments dans l'ordre de valeur nutritive décroissante suivant : blanc d'œuf $>$ œuf entier $>$ muscle de bœuf $=$ caséine $>$ farine d'arachide $>$ gluten de blé; quoique les valeurs moyennes de tous les laboratoires suivent cet ordre, il y a de grandes variations individuelles dans les résultats obtenus par différents laboratoires ; la méthode paraît reproductible seulement dans un laboratoire donné. La valeur biologique de l'œuf entier commercial Rutgers est plus basse que celle de l'échantillon préparé au laboratoire (V. B. $=86,8$ contre 97,6) et ces deux valeurs sont significativement différentes ( $\mathrm{P}$ inférieur à $0, \mathrm{OI}$ ).

Par ailleurs, Mitcheli a analysé les nombreux résultats obtenus sur la croissance du rat pour différentes protéines et a comparé ces résultats avec les index chimiques de MirchelL et Oser des protéines correspondantes. Les coefficients de corrélation entre la valeur biologique et les coefficients chimiques de Mitchell et d'Oser sont respectivement 0,86 et 0,85 ; de plus, l'analyse de la variance montre que $73 \mathrm{p}$. Ioo de la variance est contrôlée par la composition en acides aminés indispensables des protéines. Comme les erreurs expérimentales étaient faibles (MITCHELL a sélectionné les résultats), la variance fortuite ( $27 \mathrm{p}$. Ioo de la variance totale) est due à d'autres facteurs que la composition en acides aminés indispensables parmi lesquels on peut signaler :

- les vitesses différentes de libération enzymatique des acides aminés au cours de la digestion ;

- la composition du reste de la ration : nature et quantité des glucides et des lipides ;

- les temps relatifs d'ingestion des différents éléments ;

- 1'action des bactéries du tube digestif qui peuvent modifier dans 
des proportions inconnues la composition en acides aminés des protéides ingérées.

Ia première raison invoquée a été démontrée dans quelques cas particuliers : quand les aliments sont stockés pendant de longues périodes ou soumis à quelques traitements industriels (cas de la farine de coton), les protéines sont d'abord modifiées sans destruction des acides aminés constitutifs, l'activité biologique est diminuée et l'hydrolyse enzymatique in-vitro de ces produits traités comparativement à celle des produits originels montre des différences dans la vitesse de libération de certains acides aminés : arginine, histidine, lysine ; et ces différences peuvent être reliées à la diminution d'activité biologique sur la croissance du rat. Si les traitements sont poursuivis plus longtemps, les acides aminés sont alors détruits et 1'activité biologique baisse corrélativement.

En résumé, dans le cas des monogastriques, la valeur nutritive des protéines appréciées par les index chimiques considérés, est une bonne approximation de la valeur de métabolisation de cette protéine; cependant, elle néglige les facteurs digestifs qui devraient assurer un apport simultané des acides aminés constitutifs sur les lieux d'utilisation. Si les produits naturels n'ont pas été soumis à des traitements industriels, ces conditions sont en général réalisées; la méthode a d'ailleurs permis de nombreuses supplémentations dont le bien fondé a été vérifié souvent sur l'animal, ce dernier est en effet le juge ultime dans des conditions physiologiques définies. Le succès des prévisions est limité actuellement d'une part, par les méthodes d'analyse imparfaites de certains acides aminés (acides aminés soufrés en particulier) et, d'autre part, par certains facteurs peu connus de l'utilisation digestive.

\section{Ruminant.}

Le métabolisme azoté interne de ces animaux semble peu différent de celui des autres mammifères comme l'ont montré les travaux de BLAXTER sur le veau et de KLEIBER sur la vache laitière ; mais le ruminant ayant ses fonctions digestives parfaitement développées possède une flore et une faune particulièrement actives dans ses grands réservoirs gastriques : les matières azotées alimentaires sont fortement remaniées par les bactéries dont les exigences azotées sont assez réduites (ammoniac et certains acides aminés soufrés).

I,e problème revient à bien nourrir les bactéries pour alimenter convenablement 1'hôte; ceci est obtenu par un rapport adéquat des glucides solubles et membranaires aux matières azotées, le soufre' devant être présent en quantité suffisante dans 1'alimentation; il faut éviter en outre, une orientation fermentaire détruisant les protéines en excès, 
inutilisées pour la croissance bactérienne ; ce qui peut être réalisé quand elles sont relativement insolubles (SYNGE I954).

D'ailleurs, la plupart des mesures de valeur biologique effectuées sur les ruminants ont procuré des valeurs assez constantes, les variations dépendent surtout du taux protidique des rations (voir tableau VI).

TABLEAU VI

Valeur biologique de quelques protéines

pour la croissance et l'entretien de différentes espèces

\begin{tabular}{|c|c|c|c|c|}
\hline & \multicolumn{3}{|c|}{ Entretien } & \multirow{2}{*}{$\begin{array}{c}\text { Croissance } \\
\text { Rat }\end{array}$} \\
\hline & Rat adulte & Homme adulte & Chien adulte & \\
\hline 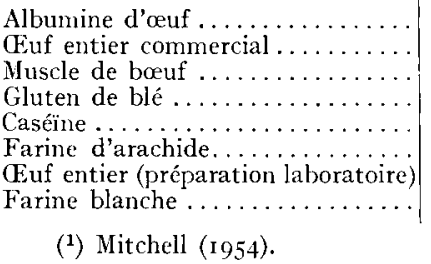 & $\begin{array}{l}94 \\
82 \\
69 \\
65 \\
51 \\
46 \\
99 \\
65\left(^{1}\right)\end{array}$ & $\begin{array}{l}91 \\
94 \\
67 \\
42 \\
56 \\
56 \\
4 \text { I (1) }\end{array}$ & $\begin{array}{l}89 \\
86 \\
57 \\
68 \\
61\end{array}$ & $\begin{array}{l}97 \\
87 \\
76 \\
40 \\
69 \\
54 \\
9^{6} \\
5^{2}\left({ }^{1}\right)\end{array}$ \\
\hline
\end{tabular}

Tableau VII

Valeur biologique pour la croissance des génisses

de diftérentes sources de matières azotées

SWANSON (r943)

\begin{tabular}{|c|c|c|}
\hline Régime & Relation nutritive & $\begin{array}{l}\text { Valeur biologique } \\
\text { pour la croissance }\end{array}$ \\
\hline Foin....... & $\frac{1}{8,5}$ & 67 \\
\hline Lait ... & . $\quad \frac{\mathrm{I}}{8,7}$ & 69 \\
\hline Foin. . & $\frac{\mathrm{I}}{\mathrm{I} 2,2}$ & $8+$ \\
\hline Lait + Foin & $\frac{\mathrm{I}}{\mathrm{II}, 5}$ & 83 \\
\hline
\end{tabular}

La valeur biologique tant pour la croissance que pour la production laitière des bovins et des ovins varie entre 60 et $65 \mathrm{p}$. Ioo si le taux protéique est optimum.

Dans le cas des ruminants le seul critère valable de la qualité de la matière azotée est la digestibilité de celle-ci très variable chez ces animaux car la matière azotée fécale dépend de la matière sèche excrétée, elle-même fonction de la quantité de glucides fibreux du régime. Sans doute pour des niveaux de nutrition très élevés une plus grande fraction des protéines alimentaires ne serait pas remaniée ce qui incluerait 1'absorp- 
tion d'une grande quantité des acides aminés alimentaires originelles; l'importance de ce phénomène est inconnue et on ne peut étendre aux ruminants ayant leurs fonctions digestives parfaitement développées ce qui a été dit du problème de la prévision de la valeur nutritive des protéines chez le monogastrique.

\section{REFÉRENCES BIBLIOGRAPHIQUES}

Black (A. L.), KLEIBER (M.) and Smith (A. H.). - J. of Biol. Chem., 19\%, 365, I952.

BlaXTer (K. L.) and Wood (W. A.). - Brit. J. of Nutr., 6, 56-7I, 1952.

Block (R. J.) and Mitchel. (H. H.). - Nut. Abst. and Rev., 16, 249, I946.

Bocobo (D. L.), Skelifenger (M.), Shaw (C. R.) and SteEle (B. F.). Arch. Biochem. Biophys., 40, 448-452, I952.

Brush (M.). - J. of Nutr., 33, 390, I947.

Cooperative determination of the amino acid content and of the nutritive value of six selected protein food sources. Bureau of Biol. Research Rutgers University, I950.

Crampton (E. W.) and Rutherford (B. E.). - J. of Nutr., 54, 445, I954. Dustin (J. R.), Schram (E.), MoOre (S.) and Bigwood (E. J.). - Bull. Soc. Chim. Biol., 35, xI37, I953.

DE Vuyst (A.). - Rev. de l'A griculture, 9, 457, I954; 10, I259, 1955.

Frazier (L. E.), BendeTt (E. P.), Woolridge (R. L.) and SteFFeE (C. M.). - J. of Nutr., 40, 335, I950.

Kuhman (J.). - Z. Lebensm. Untersuch and Forschung, 90, 434, I950.

Loosli (J. K.), Maynard (L. A.), Curtiss (L. V.) and all. - The aminoacid requirement for the growth of Swine, Cornell Univ. N. Y., I950-I955.

Mrtcheli. (H. H.) and BeAdLES (J. R.). - J. of Nut., 4\%, I33, I952.

Mitcheli, (H. H.). - P. 279. IOO Jahre Mockern die Bewertung des Futterstoffe and andere Probleme der Tierernährung, Band II, I954.

Morris (S.). - J. of Dairy Res., 4, I77, I953.

OSER (B. L.). - J. of Am. Diet. Ass., 217, 197, I95I.

Rose (W. C.) and ail. - The aminoacid requirement of man. J. Biol. Chem., I950-I955; J. Biol. Chem., 214\%, 87, I955.

Sheiton (D. C.), BeEson (W. M.), Mertz and all. - The aminoacid requirement for the growth of swine. Purdue Univers. Indiana, I95I-I955.

Swansson (E. W.) and Hriman (H. S.). - Miss. Agr. Exp. Stat. Res., bul. no 372 , I943.

Wirliams (H. H.). - Agric. Exp. Stat. Cornell Univers. N. Y., mémoire no 337 , I955. 